\title{
TOPONOMASTICA SACRA E PROFANA NELL'EGITTO GRECO-ROMANO
}

\author{
Nicola Reggiani \\ Università degli Studi di Parma, Italia
}

\section{Sacred and profane place names in Graeco-Roman Egypt}

\begin{abstract}
The papyri of Graeco-Roman Egypt provide, among numerous historical and social data, invaluable information about the linguistic context of a deeply multilingual society, where especially Egyptian (Demotic) and Greek (Hellenistic Koine of everyday use) intertwine with each other in a dialectic relationship rich in interesting causes for reflection. Papyrological sources offer a truly interesting bulk of information related to local place names, which show sacred (related to the Egyptian gods) and profane elements, often varying according to the Greek or Egyptian pronunciation of the same name. The paper outlines some general trends by presenting a selection of relevant cases.
\end{abstract}

Keywords: place names of Graeco-Roman Egypt, Greek papyri, ancient multilingualism, ancient translations.

\section{Introduzione}

La pervasività della sfera religiosa nella cultura e nella quotidianità dell'antico Egitto era chiaramente percepita dai Greci che, almeno a partire dal VII sec. a.C., erano entrati in contatto più o meno diretto con la millenaria civiltà faraonica. Fra molti possibili riferimenti, una constatazione di Erodoto è particolarmente significativa: lo storico di Alicarnasso nota (Storia II 37,1) che gli Egizi sono "straordinariamente reli-

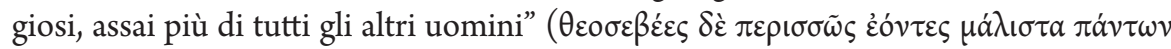
$\dot{\alpha} v \theta \rho \dot{\omega} \pi \omega v)$. Fra le manifestazioni religiose degli antichi Egizi, il ricchissimo e prevalentemente teriomorfo pantheon colpiva particolarmente (come ci colpisce tutt'oggi) la curiosità greca. Entrati in contatto con le multiformi divinità egiziane, i Greci le metabolizzarono nella propria cultura e Weltanschauung secondo diverse modalità linguistiche: (a) adattamento del teonimo egiziano in greco, attraverso la sua traslitterazione fonetica e la sua declinazione secondo le categorie grammaticali elleniche (es. Wsir [Osiride] > Osiris, İs.t [Iside] > Isis; $\mathrm{Hr}$ [Horus] > Horos; Tz-wr.t [Tauerit] > Thoeris; ecc.); (b) interpretatio Graeca, ovvero l'associazione della divinità egiziana con una corrispondente greca, assimilata in base a caratteristiche teologiche, cultuali, rituali simili (es. İmn [Amon] = Zeus, $R^{\prime}[\mathrm{Ra}]=$ Helios, $H r$ [Horus] = Apollo, $\underline{D} h w$ wy [Thoth] $=$ Hermes, $H$ w.t $-H r$ [Hathor $]=$ Aphrodite $)^{1}$; (c) sincretismo fra divinità diverse, una

\footnotetext{
1 Cf. von Lieven 2016.
} 
tendenza particolarmente favorita dai sovrani dell'Egitto tolemaico per rinsaldare la fusione fra i nuovi arrivati greci e gli indigeni egiziani (es. Oserapis/Serapis $=$ Osiris + Hapy + Zeus) $)^{2}$. Casi particolari sono piuttosto sintomatici di certe tendenze che ritroveremo nelle modalità di adattamento e traduzione dei toponimi, di cui ci occuperemo nel presente contributo: $S b k$ [Sobek], ad esempio, il notissimo dio-coccodrillo, venne reso con Souchos, che in greco indica appunto l'animale sacro al dio, oltre ad essere parzialmente omofono dell'originale egiziano.

La toponomastica dell'antico Egitto è fortemente imbevuta di valori e significati religiosi. Ogni insediamento di una certa ampiezza possedeva edifici sacri ed era votato in particolare al culto di una specifica divinità, che spesso riceveva peculiari attributi legati al toponimo stesso, come, ad esempio, il caso del Sobek venerato a Tebtunis, noto come Sbk-nb-tn [Soknebtunis] "Sobek, signore di (Teb)tunis"3. Numerose località, di contro, contenevano nel proprio nome un elemento teonomastico. Nell'esigenza, amministrativa prima ancora che culturale, di adattare alla lingua greca la toponomastica egiziana, vennero adottate diverse strategie nel trattamento dei toponimi, sia di quelli di tipo sacro, sia di quelli che invece non contenevano riferimenti religiosi. I papiri scritti in greco (e in demotico) e ritrovati in abbondanza perché preservati dalle sabbie d'Egitto costituiscono un'insostituibile fonte al proposito ${ }^{4}$. Nelle pagine successive presenterò una casistica selezionata, ma significativa, dei fenomeni di conversione toponomastica dall'egiziano al greco e (in certi casi) viceversa, basata sui dati raccolti nel catalogo Trismegistos Places 5 .

\section{Toponimi sacri}

2.A. Toponimi sacri egiziani resi in forma greca

Spesso (ma non sempre) sono caratterizzati da una prefissazione significativa (Bou- o Per/Pher- < eg. Pr "casa", nel senso di "casa della divinità", i.e. "tempio"; Kerke$<$ eg. $g r g$ "insediamento") seguita dal teonimo reso in forma greca.

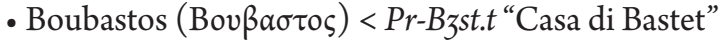

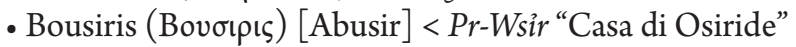

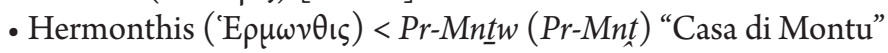

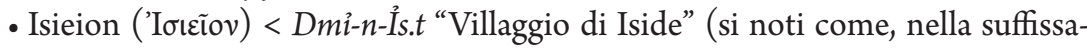
zione -ieion, il toponimo greco richiama la tipica onomastica templare greca, es. Asklepieion, Artemision, ecc.)

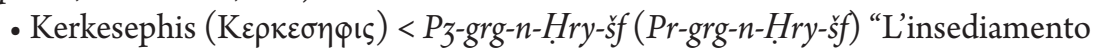
di Harsaphes"

2 Cf. Borgeaud and Volokhine 2000.

Cf. Pernigotti 1997: 165-166.

4 Si vedano ad esempio le numerose e dettagliate informazioni raccolte nel Dizionario dei nomi geografici e topografici dell'Egitto greco-romano (Calderini and Daris 1935-2010), in cinque volumi con cinque Supplementi.

5 http://www.trismegistos.org/geo (cf. Reggiani 2017: 69). Ad esso, per semplicità, si rimanda per ogni riferimento bibliografico ai toponimi citati nel prosieguo dell'articolo. 


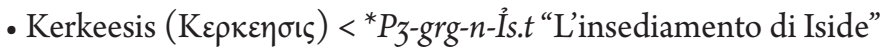

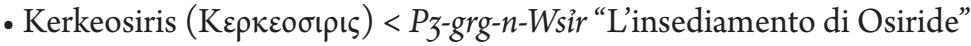
Sobek"

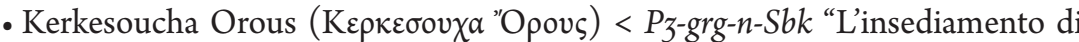

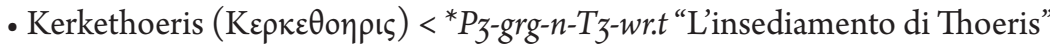

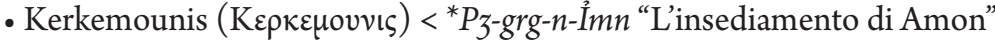

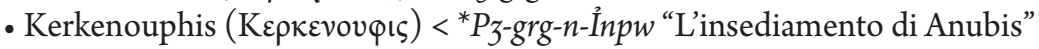

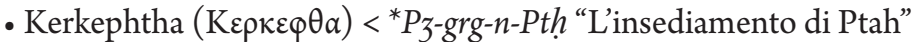

- Perkethaut $=$ Philagris $(\Pi \varepsilon \rho \kappa \varepsilon \theta \alpha v \tau=\Phi i \lambda \alpha \gamma \rho i \varsigma)<$ Pr-grg-n-Dhwty $\left({ }^{*} P\right.$ z-grg-nDhwty) "L'insediamento di Thoth"

- Pharbaithos $(\Phi \alpha \rho \beta a \imath \theta \varsigma)$ < Pr-Hr-mr.ty "Casa di Harmerty (= Horus dai Due Occhi)"

- Pseonnophris ( $\left.\Psi_{\varepsilon} o v v \omega \varphi \rho ı\right)<{ }^{*} P 3^{-?-n-W n-n f r}$ "Il ... di Onnophris (= Osiride)"

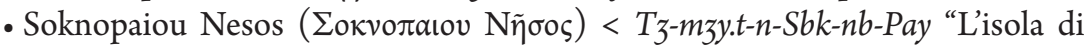
Sobek, signore di Pai"

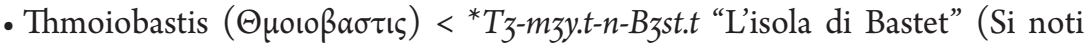
come, a differenza del toponimo precedente, dove l'elemento geografico tz-mzy.t "l'isola" viene tradotto nel corrispondente greco $v \tilde{\eta} \sigma o \varsigma$, qui esso viene semplicemente traslitterato foneticamente in thmoio-).

\section{B. Toponimi sacri egiziani resi in interpretatio Graeca}

Spesso (ma non sempre) sono caratterizzati da una suffissazione significativa (-polis "città" che rende l'egiziano pr "casa" o simili) preceduta dal teonimo reso con interpretatio Graeca.

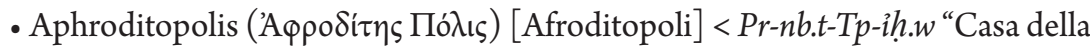
Signora della Testa di Vacca (= Hathor)", identificata con Afrodite.

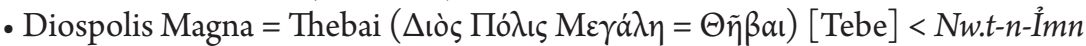
"Città di Amon" = Pz-dmi-İmn "Il villaggio di Amon", identificato con Zeus.

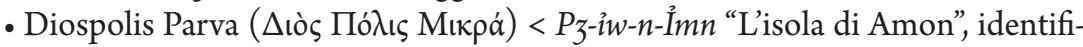
cato con Zeus.

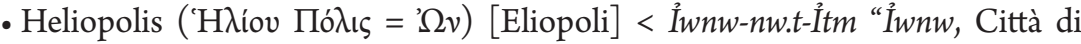
Atum" = Íwnw-R " "Iw wnw, (Città di) Ra" = Pr-R "Casa di Ra”, identificato con il Sole (Helios). ' $\Omega v$ è traslitterazione fonetica del nome 'profano' della città, İwnw.

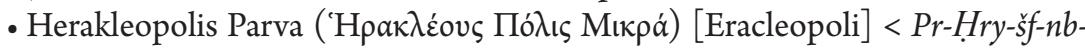
$N n$-nsw "Casa di Harsaphes, signore di Nn-nsw", identificato con Eracle.

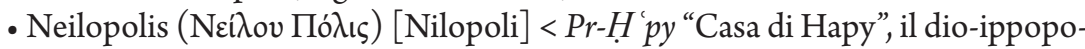
tamo associato alle piene del Nilo.

- Panopolis (Пavòs Пódıs) [Panopoli] < Hnty-Mìn “Entrata di Min”, identificato con Pan.

Due casi particolari da notare sono i nomi di Ossirinco, $\operatorname{Pr}-M \underline{d} d \underline{d}$ "Casa del Pesce Medjed" (un pesce sacro venerato in loco), reso col nome greco del pesce

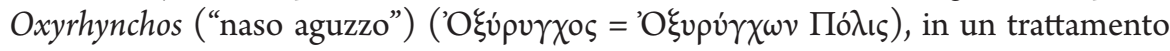




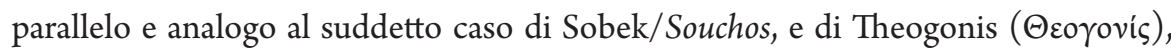
che rende in letterale traduzione l'egiziano Pz-i.ir-p3-nt $\underline{t}$ "Quello che il dio ha fatto".

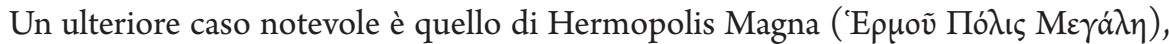
originariamente Hmnw "Gli Otto" in riferimento alla Sacra Ogdoade là venerata; uno di questi otto dèi principali era Thoth, identificato con Hermes, da cui il toponimo greco.

\section{Toponimi profani}

\section{A. Toponimi profani egiziani resi in traslitterazione fonetica}

Si notino le frequenti incertezze ortografiche nel passaggio da una lingua all'altra.

- Athribis $(A \theta \rho ı ß \varsigma)<H w . t-h r y-i \hat{b}$

- Haueris (Aúnpıs) [Hawara] < Hw.t-wr.t = Hw.t-wry "il grande palazzo"

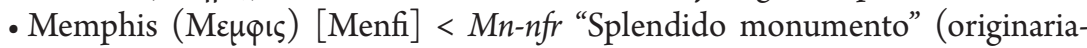
mente, la piramide di Pepi I)

- Mouchis $($ Movxı $)<P r-m h y\left(P r-m h=P z-m w h y s=P z^{-} \cdot\right.$.wy-n-Pr-mwhz? $)$ "Il posto di Per-moucha"?

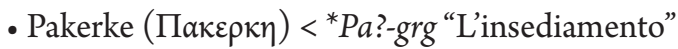

- Sais $\left.\left(\sum a ı\right)\right)<S_{3} w=S_{z} y=S y$

- Sethrempais $\left(\sum \varepsilon \theta \rho \varepsilon \mu \pi \alpha \iota \varsigma / \sum \varepsilon v \tau \rho \varepsilon \mu \pi \alpha ı \varsigma\right)<P z-s t r z-n-P a-h y$ "Il luogo di riposo di Pais"?

- Psophthis $(\Psi \omega \varphi \theta \imath \varsigma / \Sigma \omega \varphi \theta \iota \varsigma)<{ }^{*} P$ z-sbt "Il muro"

- Syene $(\Sigma v \eta v \eta / \Sigma o \eta v \eta)[$ Assuan] $<$ Swn $(S w n w)$

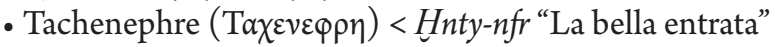

- Tanis $($ Tavıs $)<\underline{D}^{\prime} n . t\left(T^{\prime} n y\right)$

- Tebtunis $(T \varepsilon \beta \tau u v i \varsigma)<T p-t n(T p-d b n)$

- Thmouis $(\Theta$ rovis $)<T$ z-mzy.t $\left(T_{3}\right.$-mzw.t) "L'isola"

- Thraso $(\Theta \rho \alpha \sigma \omega / \Sigma \alpha \theta \rho \omega)<{ }^{*} T_{3}$-rsy (Trswz) "Area chiusa, torre di guardia"

\section{B. Toponimi profani egiziani tradotti in greco}

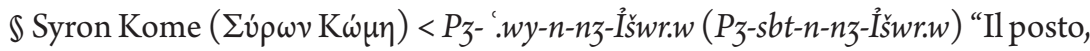
la fortezza degli Assiri”

\section{C. Neotoponimi profani greci, traslitterati o tradotti in egiziano}

I toponimi delle neofondazioni greche in Egitto sono esclusivamente profani, generalmente di tipo onorifico (ispirati a nomi o attributi dei sovrani della dinastia tolemaica) o derivanti da antroponimi ellenici (spesso di origine micrasiatica o caria, da dove provenivano i primi Greci immigrati in Egitto, o legati alla dinastia tolemaica). La loro resa in egiziano è pressoché esclusivamente fonetica.

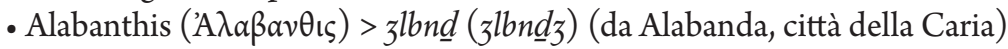

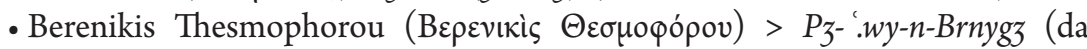
Berenike, una sovrana d'Egitto). 


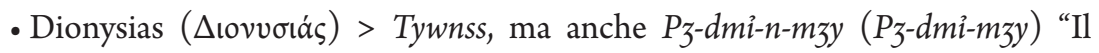
nuovo villaggio"

- Philoteris $(\Phi \imath \lambda \omega \tau \varepsilon \rho i \varsigma)>P z^{-} \cdot$.wy-n-Pltrz $\left(P z^{-}\right.$'.wy-n-Pyltr) "Il posto di Philotera" (figlia di Tolemeo I).

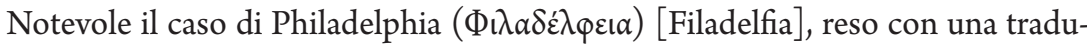
zione letterale Pz- 'wy-n-tz-mr.t-sn "Il luogo di colei che ama suo fratello" (appellativo dinastico tolemaico), ma originariamente chiamato Nz-nh.w "sicomori". Nei casi in cui la fondazione greca si sovrappone a un precedente insediamento, l'egiziano solitamente mantiene il toponimo originario, es. Alexandrou Nesos ('A $\lambda \varepsilon \xi \dot{\alpha} v \delta \rho \rho o v \mathrm{~N} \tilde{\eta} \sigma o \varsigma)$, orig. Tz-mzy.t-n-Pz-whr "L'isola di Paueris", o Lysimachis ( $\Lambda v \sigma \mu \alpha \chi i \varsigma)$, orig. Tz-s.t$n-P a-N z-d b_{3} . w$ "Il posto di Panetbeus". Nel caso della capitale d'Egitto, Alessandria ('A $\lambda \varepsilon \xi \hat{\alpha} \nu \delta \rho \varepsilon 1 \alpha)$, il toponimo dà luogo a $P z^{-}$'.wy-n-zlgszntrs ("Il luogo di Alexandros"), ma l'originario $R^{\prime}-q d\left(R^{\prime}-q t\right)=$ Rakotis ("terreno da costruire, fine delle abitazioni?") si mantiene fino alla resa copta ракотє - pдкот.

\section{Toponimi sacri $\leftrightarrow$ profani}

\section{A. Toponimi profani egiziani, sacralizzati in greco}

In alcuni casi particolari, toponimi originariamente profani vengono sacralizzati in greco, sulla base del culto principale tributato nella località.

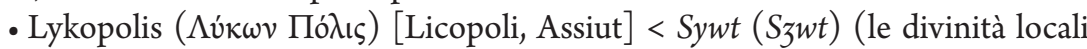
erano Anubis e Upuaut in forma di sciacallo/cane)

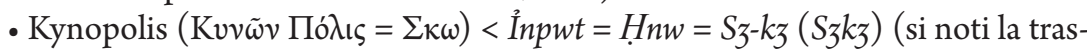
litterazione fonetica $\Sigma \kappa \omega$ ) (sede di un famosissimo culto dei cani)

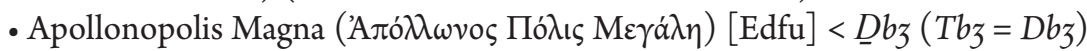
$=B h d t(B h t)=M s n$ (sede di un famosissimo culto di Horus)

\section{B. Toponimi sacri egiziani, desacralizzati in greco}

Il processo inverso al precedente si verifica in casi di paretimologie, come per

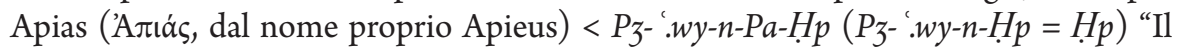
posto di Hapy", oppure in casi di traslitterazioni fonetiche che perdono il riferimento sacro originario:

- Mendes $(\mathrm{M} \varepsilon v \delta \eta \varsigma)<\operatorname{Pr}-B z-n b-\underline{D}$ d.t "Casa dell'Ariete, signore di Djedet"

- Psenhyris $(\Psi \varepsilon v v \rho ı)<{ }^{*} P z-s y-n-H r$ "Il lago di Horus"

- Sebennytos $\left(\sum \varepsilon \beta \varepsilon v v v \tau \tau o \zeta\right)<\underline{T b}$-nt $\underline{r}(T b n-n \underline{t} r=S b n-n \underline{t} r=\underline{D} d b-n-n \underline{t} r) \ll($ Città del) Vitello Divino»

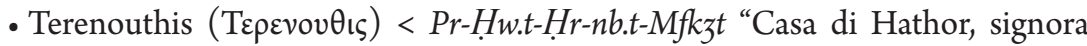
della Turchese"

Singolare il caso di Pathyris ( $₫ \alpha \theta v \rho ı)$, che traslittera l'egiziano Pr-Hw.t-Hr "La casa di Hathor", sebbene un altro nome greco di questa località mantenga l'elemento

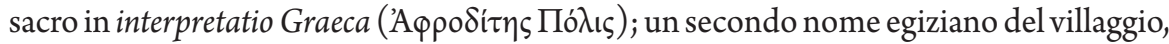

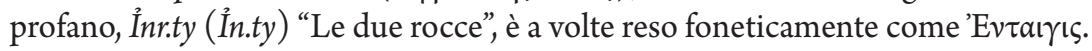




\section{Conclusioni}

Per concludere, a ideale sunto delle complesse dinamiche che animano la toponomastica greco-egiziana, osserviamo il caso della capitale del nomos Arsinoite, il distretto dell'attuale oasi del Fayum. Il toponimo egiziano profano $\check{S} d t(\check{S} t y)$, cede il posto al toponimo greco sacro Krokodilopolis (Кроко $\delta \hat{\lambda} \omega \nu$ Пó $\lambda ı$ ) in virtù dei culti locali tributati a divinità in forma di coccodrillo; ma la città ha anche nomi greci profani

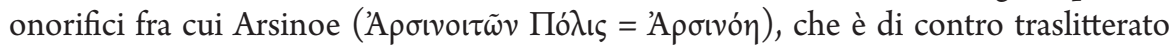
foneticamente in egiziano zrsynz. Un continuo flusso linguistico-culturale fra elemento greco ed egiziano, che rappresenta un po' la cifra dell'intera civiltà sviluppatasi dall'età ellenistica in avanti lungo il corso del Nilo.

\section{Bibliografia}

Borgeaud, P. e Y. Volokhine. 2000. La formation de la légende de Sarapis: une approche transculturelle. ARG 2: 37-76.

Calderini, A. e S. Daris (eds.). 1935-2010. Dizionario dei nomi geografici e topografici dell'Egitto greco-romano. Milano-Pisa: Goliardica-Fabrizio Serra.

Pernigotti, S. 1997. Tre Sobek del Fayyum. Simblos 2: 165-174

Reggiani, N. 2017. Digital Papyrology I. Methods, Tools and Trends. Berlin-New York: De Gruyter. Von Lieven, A. 2016. Translating Gods, Interpreting Gods. On the Mechanisms behind the Interpretatio Graeca of Egyptian Gods. In Greco-Egyptian Interactions: Literature, Translation, and Culture, 500 BC - AD 300, I. Rutherford (ed.), 61-82. Oxford: Oxford University Press. 